\title{
Promoting Health for Chronic Conditions: a Novel Approach that integrates Clinical and Personal Decision Support
}

\author{
I. Lasorsa*, M. Ajčević, P. D’Antrassi ${ }^{*}$, G. Carlini ${ }^{* *}$, A. Accardo* and S. Marceglia* \\ * Department of Engineering and Architecture, University of Trieste, Trieste, Italy \\ ${ }^{* *}$ R\&D Department, TBS Group, Area Science Park, Trieste, Italy \\ irene.lasorsa@phd.units.it
}

\begin{abstract}
Direct and indirect economic costs related to chronic diseases are increasing in Europe due to the aging of population. One of the most challenging goals is to improve the quality of life of patients affected by chronic conditions, and enhance their self-management. In this paper, we propose a novel architecture of a scalable solution, based on mobile tools, aimed to keep patients with chronic diseases away from acute episodes, to improve their quality of life and, consequently, to reduce their economic impact. Our solution aims to provide patients with a personalized tool for improving self-management, and it supports both patients and clinicians in decision-making through the implementation of two different Decision Support Systems. Moreover, the proposed architecture takes into account the interoperability and, particularly, the compliance with data transfer protocols (e.g., BT4/LE, ANT+, ISO/IEEE 11073) to ensure integration with existing devices, and with the semantic web approaches and standards related to the content and structure of the information (e.g., HL7, ICD-10 and openEHR) to ensure correct sharing of information with hospital information systems, and classification of patient behaviors (Coelition). The solution will be implemented and validated in future study.
\end{abstract}

\section{INTRODUCTION}

In the European region, the population aged 65 and over is projected to rise from $15 \%$ in 2000 to $23.5 \%$ by 2030 [1]. This trend is considered as one of the reasons for the growing burden of chronic conditions and diseases. Chronic diseases, such as coronary heart disease, chronic respiratory diseases and diabetes, are the leading cause of mortality and morbidity in Europe [2]. The home environment and the informal caregivers are the most common care solution for chronic patients. The interaction among patients/caregivers and healthcare providers needs to be strengthened to enhance adherence to the clinical pathway and monitor health outcomes.

Despite the increasing burden of chronic illnesses, healthcare is still largely built around an acute, episodic model of care that is ill-equipped to meet the requirements of those with chronic health problems [3]. Mobile technologies can support a healthcare redesign based on disease management programs and integrated care models [4], [5], [6], [7].
Mobile technologies may act as a complementary tool to provide support and motivation to regular patient's selfmonitoring of health parameters (e.g., blood pressure, heart rate, blood glucose) [8]. The resulting empowerment may help care recipients to achieve personal health objectives, modify lifestyle patterns and/or high-risk behaviors for an optimal management of their chronic condition.

At present, over 35,000 mobile health (mHealth) apps are available for iOS and Android [9], but, despite the widespread diffusion, a lot of challenges are still to be faced, such as apps security, data safety, regulatory framework, interoperability, certification and quality, and integration in existing healthcare models [10], [11].

In the last decades, Decision Support Systems (DSSs), defined as any electronic or non-electronic system designed to aid directly in clinical decision making [12], gained increasing attention thanks to the promise to use characteristics of individual patients to generate patientspecific assessments to support clinicians' decisionmaking.

On the other hand, even though mobile technologies can ground the development of patients' self-management systems, the development and use of DSSs for supporting patients' decision-making is still lacking.

In this paper, we preliminarily present the architecture of a comprehensive, mobile-based, patient-oriented solution aimed to diminish acute episodes of patients with chronic diseases in order to reduce their economic impact on the healthcare system and to improve their quality of life. These goals can be reached through the development of a new model of relation between patient and disease, based on a mobile technology that integrates Clinical DSS (CDSS) for physicians and Lifestyle DSS (LDSS) for patients. The model is primarily focused on selfmanagement of care, instead of handling the mere information exchange between patients and healthcare professionals.

\section{REQUIREMENTS}

Clinical physician-oriented DSSs are largely used and implemented while the new challenge is to create and integrate a patient-oriented DSS as an innovative tool 
designed with the aim of providing patients with more knowledge about their conditions and the disease they are affected by, and guide them towards a healthy lifestyle.

The majority of the mHealth existing solutions (e.g., glooko, bloodpressurecompanion, moticon, Metria ${ }^{\mathrm{TM}}$

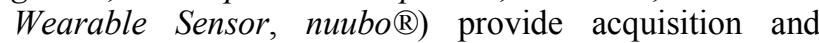
processing of pathology related to biomedical data and health status. However only few of them (e.g., mySugr, Indi $G O^{\mathrm{TM}}$ ) provide integration with clinical evidence and guidelines, and prediction of health status.

The most common mHealth solution state-of-art limitations concern:

- Lack of interoperability and scalability which also result in high initial costs for citizens [13];

- Lack of standards: many systems work only on proprietary platforms, from sensors to software (e.g., iPhone) and integrations with open platform such as Android are limited [14], [15];

- Cultural challenge: patients are unaccustomed to use technologies for clinical purposes, as they usually prefer to contact doctors directly [16];

- Need to improve mobile interface userfriendliness and user experience [17];

- Need to increase the mutual information flow between healthcare professionals and patients rather than keeping with one direction flow of data from patients to clinicians [16];

- Need to guarantee privacy, integrity and confidentiality of the system information.

The novelty of our proposal mainly concerns the requirements identified as key features of our system, that overcome the aforementioned limitations of the solutions currently available in the literature and market. Particularly, we defined the following requirements:

- Open architecture;

- Coexistence of clinical and lifestyle decision support systems;
- Compliance to the existing standards and regulations;

- Scalability and versatility of the system customizable to patients affected by different chronic diseases.

\section{ARCHITECTURE}

The proposed architecture is based on three layers (i.e., patient self-management, multiservice storage system and clinician supervision), as shown in Fig. 1.

In order to allow the customization for different needs of different user groups (e.g., pathology, age) the use of loosely coupled set of heterogeneous modules were used. These modules are organized as apps, services and processes running either in foreground or background according to the specific implemented functionality. Regarding the scalability, our proposal is an architectural paradigm able to sustain a system designed to seamlessly handle changes and reconfigurations both in its front-end and in its back-end (Fig.1). Hence, introducing new sensors or apps in the input space or rules in the back-end that lead to new users' functionalities (in the form of apps and tools) available at the output space, requires only minimal intervention. In order to comply with the privacy requirements, no sensitive clinical data are stored on the mobile device: personal, sensors and therapy information are stored in a remote DB that ensures privacy and provides a highly concentrated source of data, shared by all the mobile SW modules. Data integrity is foreseen through algorithms that can assure that authenticate users are handling unchanged and original information (cryptographic hash functions, such as Message Digest 5 or Secure Hash Algorithm 1). To ensure confidentiality of data exchanged through the network from unauthorized/authorized agents, our solution is based on an encryption/decryption hybrid approach using symmetric and asymmetric algorithms to guarantee data confidentiality between the communication processes.

The patient is engaged in the self-management starting from data acquisition using biomedical wireless sensors and also through input of self-reported data.

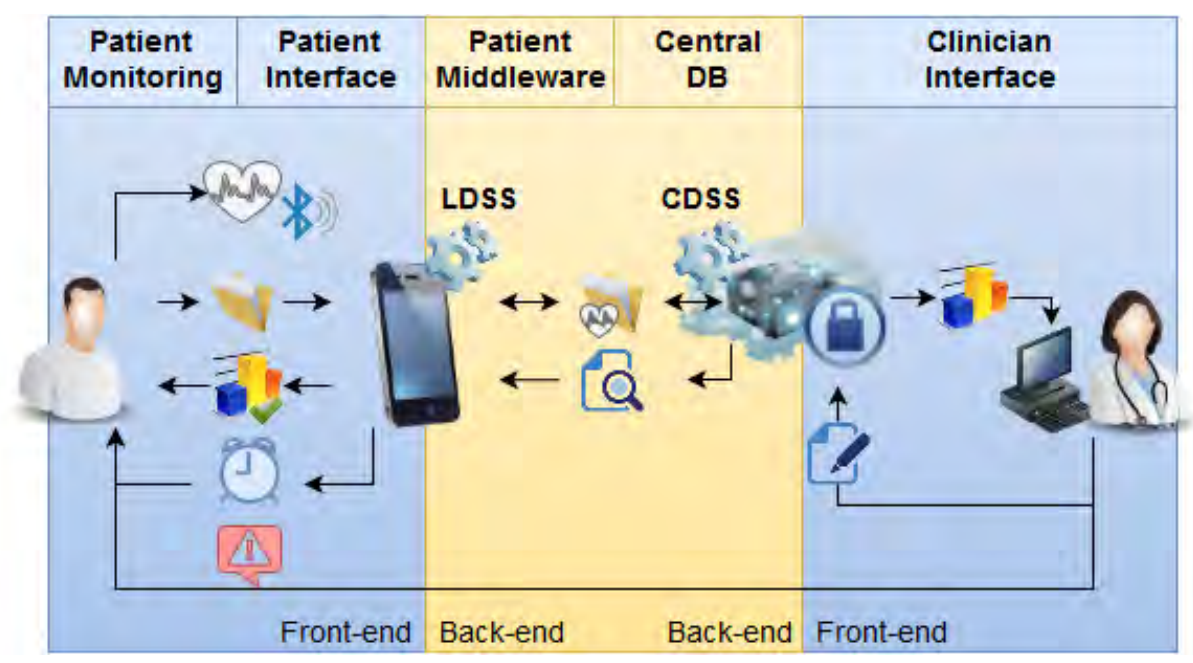

Figure 1. Overview of the proposed architecture 
The collected data are processed firstly in the mobile device where they are validated, sent and, subsequently, stored in a central DB. The stored data (i.e., acquired new data, historical data, therapy, etc.) are extracted to feed a LDSS inference engine in order to provide feedback, recommendations and therapy reminders to the patient. Furthermore, interfaced with central DB, a CDSS provides a diagnostic and prognostic support to the clinician accessing the platform through a dedicated portal. The physician can directly alert the patient in emergency cases or change the prescribed therapy.

The access to the central DB requires basic authentication, SSL channel and information cryptography. For the content and structure of the information semantic, web approaches and standards (e.g., HL7, ICD-10, and OpenEHR) are used to ensure correct sharing of information with hospital information system, and classification of patient behaviors (Coelition).

The components of the architecture are briefly detailed in the following sessions.

\section{A. Patient Monitoring}

The first layer of the platform consists of a sensing device network which feeds the system with patient biomedical data needed for patient monitoring. Heterogeneous pathologies require different modalities/sensor types (e.g., weighing scale, blood pressure monitor). Consequently, the mobile application considers the compliance with transfer and data protocols (e.g., BT4/LE, ANT+, ISO/IEEE11073) to ensure integration with existing wearable devices.

\section{B. Patient Interface}

The patient interacts with the mobile application through its user-friendly interface, combining various input and output modalities (such as seeing/vision, hearing/audition, haptic/tactile). The main functionalities of the interface are:

- displaying questions to the patient;

- receiving feedback from the patient;

- providing push messages (i.e., alerts, lifestyle suggestions, reminders, achieved goals);

- enhance patients' involvement, engagement and awareness through smart design and interaction, and social networking;

- displaying biomedical parameters.

\section{LDSS: Patient's Lifestyle DSS}

The LDSS correlates processed and validated data, coming from the central DB (from questionnaires, biomedical data, etc.) and lifestyle suggestions. This DSS provides patients only with recommendation related to healthy behaviors. It supports patients for staying away from acute episodes of their chronic condition through lifestyle suggestions.

The core of LDSS is represented by an inference engine based on principles of probabilistic graphical models. For each specific condition addressed (e.g.,
Diabetes, Coronary Heart Disease CHD, Chronic Obstructive Pulmonary Disease COPD and Chronic Hypertension CHTN) a dedicated probabilistic model is implemented considering guidelines and existing predictive models.

The LDSS uses information stored on the central DB in order to provide customized advices related to the management of the patient's conditions. Particularly, the LDSS sends periodic advices concerning one or more of the following:

- identification of undesired trends in measurements;

- advice on healthy lifestyle including physical activities and suggested exercises;

- dietary advice;

- reminders regarding medications.

The LDSS knowledge base is implemented using the Unified Medical Language System (UMLS) to represent concepts and relationships.

\section{Patient Middleware}

The previous modules are orchestrated around a middleware layer, conceived as a bus acting as the glue of the mutually interacting app(s), services and processes of the patient side architecture. The middleware offers all modules abstraction mechanisms to provide a generic API, suitable for the most common needs for customer extensions or third party apps and process integration, so that other mobile embeddable devices can be integrated directly onto the patient framework. Its main purpose is to direct and integrate processes and services. Table 1 shows the core capabilities provided by the middleware bus layers and it is also capable to proxy third party devices operative paradigms into our execution model for input data management and data conversion.

\section{E. Central DB}

The central DB is a database located in a separate and secure data center that provides a highly concentrated source of data and reports of randomized controlled patient's lifestyle. Records contain the biomedical information and preferences of the patient collected by the LDSS and the related therapy and suggestions provided by the clinician through the CDSS.

According to the Service-Oriented Architecture (SOA), the external database comes with a web service on server side in order to manage data flows with the central DB. An Input Data Manager Tool categorizes data in functional structures and stores them in the mobile DB of the device as RAW data. The latter are collected and processed by DSS in order to extract parameters and write them on the DB. Thus, the DB represents the source of data for all the tools integrated in middleware.

One of the key features in the pre-processing stage is the pre-validation of the biomedical data: if the measurements are out of predetermined safe ranges, defined according to different and specific situations, the system asks the patient to repeat the measurement. 
TABLE I.

\section{F. CDSS: Clinical DSS}

The CDSS provides automated advices to healthcare professionals by constantly monitoring and interpreting the patients' data. This goal is achieved by using an intelligent diagnostic system based on existing probabilistic models (e.g., Archimedes [18], EAGLE [19], ADA guidelines [20], Seattle model [21], MAGGIC [22], ESC guidelines) that, according to the peculiarities of each patient, identifies trends and indicates possible undesired conditions or worsening of patient's health.

The CDSS provides advices intended for healthcare professionals. It is responsibility of the professional caregiver to interpret the output generated by the CDSS and decide whether to take further actions or ignore the alert provided by the CDSS. If the caregiver decides to take further actions, this is done by sending feedback (i.e., push messages) through the ICT network to the LDSS or contacting directly patients through SMS or phone calls.

\section{G. Clinician Interface}

The Clinician Interface foresees different User Profiles (Operators, Nurse, General Practitioner, and Specialist) that follow different rules in order to remotely monitor the patient by mean of several tools, such as:

- Alarm Monitor

- Electronic Health Record

- Signal Visualization

- Diagnostic Tools (advanced signal processing)

- Notifications/Lifestyle Advice

The Clinician Interface is developed in accordance to Medical Device Directive compliance.

\section{DISCUSSION}

Here we proposed a novel architecture based on mHealth technologies for improving quality of life of patients affected by chronic conditions, and empowering their self-management. The architecture's requirements are thought to overcome the main limitations of the solutions currently available in the literature and on the market. The modular architecture allows the technology to be customizable, scalable and applicable to different kinds of chronicity. The proposed architecture is based on the standards that guarantee interoperability with other medical devices and users' privacy. Furthermore, the integration of two different Decision Support Systems, one dedicated to patients and one to clinicians, helps both patients and clinicians in decision-making for a better management of chronic condition oriented to decrease acute episodes.

Our system may improve and enhance the communication and information flow between patients and medical professionals through the direct involvement of clinicians in case of need.

However, in order to further boost patients' wellbeing and engagement, the system may support both patients' compliance with a good self-management and a positive mental status. Indeed, even though the DSS can improve

\begin{tabular}{|c|c|}
\hline Capability & Description \\
\hline Invocation & $\begin{array}{l}\text { support for synchronous and asynchronous } \\
\text { communication protocols, service mapping } \\
\text { (locating and binding) }\end{array}$ \\
\hline Routing & $\begin{array}{l}\text { addressability, content-based routing, rules- } \\
\text { based routing, policy-based routing }\end{array}$ \\
\hline $\begin{array}{l}\text { Mediation \& } \\
\text { Protocol } \\
\text { Conversion }\end{array}$ & $\begin{array}{l}\text { adapters, protocol transformation, service } \\
\text { mapping, as we are expecting to integrate } \\
\text { different components, with likely different } \\
\text { origins and thus semantics }\end{array}$ \\
\hline Messaging & $\begin{array}{l}\text { message processing, message transformation and } \\
\text { message enhancement }\end{array}$ \\
\hline $\begin{array}{l}\text { Process } \\
\text { choreography }\end{array}$ & implementation of complex business processes \\
\hline $\begin{array}{l}\text { Service } \\
\text { orchestration }\end{array}$ & $\begin{array}{l}\text { coordination of multiple implementation services } \\
\text { exposed as a single, aggregate service }\end{array}$ \\
\hline $\begin{array}{l}\text { Complex event } \\
\text { processing }\end{array}$ & $\begin{array}{l}\text { event interpretation, correlation, pattern } \\
\text { matching }\end{array}$ \\
\hline $\begin{array}{l}\text { Quality of } \\
\text { service }\end{array}$ & $\begin{array}{l}\text { security (encryption and signing), reliable } \\
\text { delivery, transaction management }\end{array}$ \\
\hline Management & $\begin{array}{l}\text { monitoring, audit, logging, metering, admin } \\
\text { console }\end{array}$ \\
\hline $\begin{array}{l}\text { Protocol } \\
\text { Conversion }\end{array}$ & $\begin{array}{l}\text { comprehensive support for topical } \\
\text { communication protocols service standards }\end{array}$ \\
\hline Security & $\begin{array}{l}\text { a standardized security model to authorize, } \\
\text { authenticate and audit use of the ESB }\end{array}$ \\
\hline Transformation & $\begin{array}{l}\text { facilitation of the transformation of data formats } \\
\text { and values, including transformation services } \\
\text { (often via XSLT or Query) between the formats } \\
\text { of the sending application and the receiving } \\
\text { application }\end{array}$ \\
\hline Validation & $\begin{array}{l}\text { validation against schemas for sending and } \\
\text { receiving messages }\end{array}$ \\
\hline Abstraction & $\begin{array}{l}\text { the provision of a unified abstraction across } \\
\text { multiple layers }\end{array}$ \\
\hline $\begin{array}{l}\text { Routing and } \\
\text { Transformation }\end{array}$ & $\begin{array}{l}\text { routing or transforming messages conditionally, } \\
\text { based on a non-centralized policy (without the } \\
\text { need for a central rules engine) }\end{array}$ \\
\hline $\begin{array}{l}\text { Queuing and } \\
\text { staging }\end{array}$ & $\begin{array}{l}\text { queuing, holding messages if } \\
\text { applications/network connections temporarily } \\
\text { become unavailable or work at different speeds }\end{array}$ \\
\hline $\begin{array}{l}\text { Commodity } \\
\text { Services }\end{array}$ & $\begin{array}{l}\text { provisioning of commonly used functionality as } \\
\text { shared services depending on context }\end{array}$ \\
\hline
\end{tabular}

the compliance of patients with their clinical pathway, an additional technique (i.e., gamification), could be added for strengthening the link between users and their good health. Moreover, an additional approach for enhancing a positive psychological status (i.e., mindfulness) should be taken into consideration in order to improve patients' quality of life.

The proposed solution will be implemented and validated in future work, also adding further features such as gamification and mindfulness.

\section{REFERENCES}

[1] J. Pomerleau, C. Knai , and E. Nolte, "The burden of chronic disease in Europe," in Caring for people with chronic disease. A health system perspective, E. Nolte and M. McKee, Eds. Maidenhead: McGraw-Hill Education, 2008, pp. 15-42.

[2] R. Busse R, Tackling chronic disease in Europe: strategies, interventions and challenges, No. 20, WHO Regional Office Europe, 2010.

[3] M. M. Harbers and P. W. Achterberg, Europeans of retirement age: chronic diseases and economic activity, Bilthoven: RIVM, 2012.

[4] R. Istepanian , S. Laxminarayan, and C. S. Pattichis "Introduction to mobile m-health systems," in M-health: Emerging Mobile Health Systems, Springer, Berlin Heidelberg, 2006, p. 3. 
[5] M. Kay , J. Santos, and M. Takane mHealth: New horizons for health through mobile technologies, World Health Organization, 2011, pp. 66-71.

[6] R. Luanrattana, K. T. Win, J. Fulcher, and D. Iverson, "Mobile Technology Use in Medical Education," Journal of Medical Systems, vol. 36, pp. 113-122, 2012.

[7] Y. SC, "Mobile applications and 4G wireless networks: A framework for analysis," Campus-Wide Information Systems, vol. 29, pp. 344-357, 2012.

[8] S. Marceglia, S. Bonacina, V. Zaccaria, C. Pagliari, and F. Pinciroli, "How might the iPad change healthcare?," J R Soc Med, vol. 105, pp. 233-41, 2012.

[9] A. Sunyaev, T. Dehling, P. L. Taylor, and K. D. Mandl, "Availability and quality of mobile health app privacy policies," Journal of the American Medical Informatics Association, amiajnl-2013, 2014.

[10] The PLOS Medicine Editors, "A reality checkpoint for mobile health: three challenges to overcome," PLoS Med, vol. 10, e1001395, 2013.

[11] P. Quinn, A.K. Habbig, E. Mantovani, and P. De Hert, "The data protection and medical device frameworks-obstacles to the deployment of mHealth across Europe?," European journal of health law, vol. 20, pp. 185-204, 2013.

[12] K. Kawamoto, C. A. Houlihan, E. A. Balas, and D. F. Lobach, "Improving clinical practice using clinical decision support systems: a systematic review of trials to identify features critical to success," Bmj, vol 330, pp. 765, 2005.

[13] Mobile Health Alliance (2013) at http://mhealthalliance.org.

[14] D. Lou, X. Chen, Z. Zhao,Y. Xuan, Z. Xu, H. Jin, et al., “A Wireless Health Monitoring System based on Android Operating System," IERI Procedia, vol. 4, pp. 208-215, 2013.
[15] B. Martínez-Pérez, I. De la Torre-Díez, and M. López-Coronado, "Mobile Health Applications for the Most Prevalent Conditions by the World Health Organization: Review and Analysis," Journal of medical Internet research, vol 15, e120, 2013.

[16] R. Elangovan, and S. Arulchelvan, "A study on the role of mobile phone communication in tuberculosis DOTS treatment," Indian journal of community medicine, vol. 38, pp. 229-233, 2013.

[17] O.Vélez, P. Okyere, S. A. Kanter, and S. Bakken, "A usability study of a mobile health application for rural Ghanaian Midwives," Journal of Midwifery \& Women's Health, vol. 59, pp. 184-191, 2014.

[18] D. M. Eddy, and L. Schlessinger, "Archimedes: a trial-validated model of diabetes," Diabetes Care, vol. 26, pp. 3093-3101, 2003.

[19] E. Mueller, S. Maxion-Bergemann, D. Gultyaev, S. Walzer, N. Freemantle, C. Mathieu, et al., "Development and validation of the Economic Assessment of Glycemic Control and Long-Term Effects of diabetes (EAGLE) model," Diabetes technology \& therapeutics, vol. 8, pp. 219-236, 2006.

[20] American Diabetes Association, "Standards of medical care in diabetes-2016 Abridged for primary care providers," Clinical Diabetes, vol. 34, pp. 3-21, 2016.

[21] W.C. Levy, D. Mozaffarian, D.T. Linker, S.C. Sutradhar, S.D Anker, A.B. Cropp, et al., "The seattle heart failure model: Prediction of survival in heart failure," Circulation, vol. 113, pp. 1424-1433, 2006.

[22] S.J. Pocock, C.A. Ariti, J.J. McMurray, A. Maggioni, L. Køber, I.B. Squire, et al., "Predicting survival in heart failure: a risk score based on 39372 patients from 30 studies," European heart journal, ehs337, 2012 